\title{
POUR UNE ANALYSE CONTRASTIVE DES TYPES DE DISCOURS: L'EXEMPLE DU FAIRE-PART DE DÉCÈS EN FRANÇAIS ET EN ESPAGNOL
}

\begin{abstract}
A bstract. Drescher Martina, Pour une analyse contrastive des types de discours: l'exemple du faire-part de décès en français et en espagnol [Towards a comparative analysis of discourse on the example of French and Spanish obituaries]. Studia Romanica Posnaniensia. Adam Mickiewicz University Press, Poznań, vol. XXVI/XXVI: 2000, pp. 67-80. ISBN 83-232-0965-0. ISSN 0137-2475.

This article undertakes the problems of contrastive analysis of discourse. The author discusses functioning of a definite, ossified structure on the example of French and Spanish obituaries. Similarities and differences in the social functioning of this type of texts are discussed in order to draw conclusions which also pertain to the theory of translation.
\end{abstract}

\section{INTRODUCTION}

Dans cette communication je me propose de plaider pour une étude contrastive des types de discours qui intègre à la fois la réflexion théorique et l'analyse empirique. Un tel projet se situe dans le cadre d'une approche que Spillner (1981) appelle 'textologie contrastive'. Ici, il m'importe d'accentuer la pertinence de la perspective contrastive pour la description des types de discours et de faire quelques suggestions théoriques et méthodologiques qui pourraient servir de base à une telle recherche. Dans la deuxième partie de mon exposé $\mathrm{j}$ 'exemplifierai ces propos par une analyse empirique du faire-part de décès français et espagnol.

\section{FONDEMENTS THÉORIQUES ET MÉTHODOLOGIQUES D'UNE ANALYSE CONTRASTIVE DES TYPES DE DICOURS}

J'emploie ici le terme de 'type de discours' ou son synonyme 'genre discursif' dans un sens préthéorique proche de son usage dans le langage quotidien. Par 'types de discours' j'entends des schémas conventionnels qui guident la production et la 
réception d'une activité langagière complexe et qui - à l'intérieur d'une communauté linguistique donnée - peuvent être soumis à des variations historiques, régionales, etc.

Chaque type de discours représente un assemblage spécifique de traits situationnels, fonctionnels et structurels. La classification traditionnelle des types de discours se base essentiellement sur les propriétés situationnelles et/ou fonctionnelles au détriment des caractéristiques structurelles. Un genre discursif représenté dans plusieurs communautés linguistiques apparaît généralement dans une situation comparable et avec une finalité communicative similaire. Par contre, sa réalisation textuelle peut être soumise à des conventions tout à fait différentes. Aussi, lorsqu'on procède à une analyse contrastive des types de discours, ce sont avant tout leurs propriétés structuro-formulatives qui méritent l'attention. En plus, et c'est ce que j'espère pouvoir montrer, une connaissance approfondie des ces traits 'de surface' contribue à une meilleure appréhension de la fonction communicative, fixée souvent de manière intuitive et sans examen exact de la structure. Dans les paragraphes suivants je développerai ces aspects en discutant d'abord quelques difficultés méthodologiques de l'analyse contrastive. Ensuite, je poursuivrai avec un bref aperçu du cadre théorique.

Lors de la comparaison de types de discours se pose avant tout le problème de l'invariant. Qu'est-ce qui nous permet de poser que deux types de discours issus de communautés linguistiques différentes sont équivalents? Qu'est-ce qu'ils ont en commun? Et comment pouvons-nous y accéder? La 'textologie contrastive' a développé plusieurs procédés pour comparer des textes appartenant à des langues différentes. Parmi ceux-là je mentionne seulement la comparaison de traductions et l'analyse de textes parallèles. Pendant que la comparaison de traductions se base sur un même texte disponible dans les deux langues, l'analyse de textes parallèles travaille avec des textes appartenant à deux langues différentes et se ressemblant sous des aspects thématiques et pragmatiques. Il s'agit donc d'une comparaison qui part de l'équivalence situatio-fonctionnelle des textes mis en relation. Les traits 'externes' d'un type de discours servent d'invariant qui permet à son tour de relier des formes interlinguales divergentes.

Cette façon de procéder connaît cependant certains problèmes. On peut bien sûr supposer que la présence de tâches communicatives à peu près égales dans deux communautés linguistiques distinctes mène à la formation de types de discours similaires. Leur comparaison repose néanmoins sur un postulat d'équivalence qui précède l'analyse empirique. Il s'agît d'une hypothèse qui n'a d'abord qu'une valeur heuristique. C'est là une différence fondamentale par rapport aux typologies de textes développées de manière déductive où les analyses empiriques ne servent qu'à confirmer des catégories préétablies.

Une étude contrastive de types de discours part donc de l'invariance de traits externes et vise à la comparaison de traits internes variables, notamment des schémas de structuration et de formulation spécifiques aux deux langues en question. Ces schémas se manifestent de façon particulièrement nette lorsque on examine des types de discours qui sont liés à des situations de communication largement standardisées et 
qui poursuivent un but communicatif très clair comme par exemple le faire-part de décès, le bulletin de météo, la recette de cuisine ou l'offre d'emploi. Ces textes utilitaires appartiennent à des genres discursifs hautement conventionnalisés et suivent, voire reproduisent, des modèles structuraux déterminés, c'est-à-dire qu'ils se composent d'éléments préfabriqués. Ils répondent à des tâches communicatives fréquentes et sont guidés par des routines qui suspendent largement la production textuelle individuelle. Sur un plan théorique, l'existence de ces genres discursifs fortement conventionnalisés met en doute l'hypothèse avancée notamment par Antos (1982:119 sq.) selon laquelle chaque texte constituerait dans sa structure de surface une réalisation unique. Ces types de discours suivent, au contraire, un schéma largement prévisible qui comprend un inventaire de composants textuels ainsi que des règles séquentielles plus ou moins contraignantes. Pour chaque composant textuel il existe généralement une série de formulations préfabriquées.

Dans la description de ces routines langagières complexes se rencontrent deux traditions linguistiques différentes: les recherches sur les types de discours, d'un côté, et une phraséologie ouverte à des aspects pragmatiques et textuels, d'un autre côté. Pour une phraséologie au sens large, telle que je l'entends ici, ce ne sont plus l'idiomaticité d'une structure, sa signification globale ou métaphorique qui constituent les critères définitoires du phrasème, mais la stabilité due au lien avec une situation de communication spécifique. Cela entraîne une modification double du concept traditionnel de phrasème qui est à la fois étendu à des unités textuelles et réduit au trait de stabilité ou reproductibilité. Une telle modification apparaît déjà chez Coulmas (1981) qui intègre les 'routines discursives' au domaine de la phraséologie. Elle est ensuite précisée et développée dans des travaux sur le langage formulaire, notamment chez Guilich (1997) et Stein (1995).

Stein néglige les recherches portant sur les types de discours et part du cadre théorique de la phraséologie. Il fait la distinction entre routines linguistiques et conceptuelles. Les routines conceptuelles concernent le niveau macrostructurel; elles déterminent la nature et l'ordre séquentiel des composants textuels. Il s'agit de suites événementielles et d'actions schématisées qui se recoupent largement avec les schémas textuels relevés dans les travaux sur les types de discours. Les routines linguistiques, par contre, concernent des unités lexicalisées complexes, c'est-à-dire des formulations préfabriquées. Si et la composition et la formulation d'un texte sont guidées par des routines, il en résulte un 'texte schématique' (musterhaft). Si, en plus, ces routines sont hautement conventionnalisées le texte tiendra du formulaire (formelhaft). Ces textes-formulaires comportent une structure textuelle préétablie avec des cases vides qui seront complétées par des contenus plus ou moins typés. Le fait de rattacher la discussion sur les types de discours à des recherches phraséologiques comme le fait par exemple Gülich (1997) - apporte à mon avis cieux avantages pour la recherche contrastive: premièrement, l'analyse s'oriente davantage sur les propriétés structurelles des types de discours et deuxièmement, elle accentue d'emblée leur stabilité et partant le caractère reproductif. 


\section{ANALYSE CONTRASTIVE DU FAIRE-PART DE DÉCÈS FRANÇAIS ET ESPAGNOL}

Je me propose maintenant de concrétiser les réflexions précédentes concernant le cadre théorique et méthodologique d'une approche contrastive des types de discours à l'aide d'un cas particulier - le faire-part de décès - dont j'examinerai les réalisations française et espagnole ${ }^{1}$. Pour des raisons pratiques liées surtout à la présentation de mes analyses j'ai choisi un type de discours assez concis, avec une flnalité relativement claire et une réalisation qui fait usage de multiples éléments préfabriqués. Mais cette approche se prête également à l'analyse de types de discours plus complexes et moins standardisés.

La comparaison de faire-part de décès provenant de différentes régions à l'intérieur des communautés linguistiques française ou espagnole révèle que les conventions structurelles et formulatives qui guident la production de ce type de discours dépendent moins d'une aire linguistique que d'une aire culturelle. Ainsi les faire-part de décès québécois, wallon et romand se distinguent parfois considérablement du modèle en usage en France. Il est de même pour les faire-part de décès hispanophones. C'est pourquoi, dans l'analyse contrastive qui suit, je me suis limitée expressément à des exemples tirés de journaux français et espagnols et j'y ai privilégié la presse nationale. L'analyse repose sur un corpus de 30 faire-part de décès espagnols et 56 faire-part de décès français qui proviennent des journaux «Le Monde», «Le Figaro», «El País», «Diario 16», «ABC» et «Levante». J'ai retenu uniquement des faire-part de décès publiés par des particuliers, c'est-à-dire des annonces dont la responsabilité incombe à des membres de la famille ou des amis du défunt. Les faire-part analysés datent des années 1993 à 1998.

L'analyse suit les trois étapes suivantes: Dans le cadre d'une comparaison intralinguale des différents textes j'ai d'abord reconstruit le modèle structuro-formulatif des faire-part de décès français et espagnols. Ce n'est qu'après que la comparaison interlinguale qui oppose les modèles spécifiques français et espagnol devient possible. L'analyse contrastive proprement dite opère donc à un niveau d'abstraction plus élevé puisqu'elle compare les modèles intralinguaux respectifs. La comparaison porte avant tout sur la dimension structuro-formulative, c'est-à-dire qu'elle cherche à déterminer l'inventaire respectif des composants textuels, leur statut séquentiel ainsi que les formulations préfabriquées utilisées lors de la mise en mots. Une dernière remarque concernant la démarche méthodologique: j'ai évidemment tenu compte de la variation entre les différents faire-part pour arriver au modèle intralingual, mais j'ai négligé les annonces qui s'en écartaient nettement. Ces textes sont certes d'une grande valeur heuristique puisque la rupture avec les conventions permet justement, par la négative, de mieux saisir le modèle en vigueur. Parmi les textes examinés ces

${ }^{1}$ Cf. K. Reiß (1977/78) pour une première approche contrastive de ce type de discours. Von der Lage-Müller (1995) présente une analyse différenciée des faire-part de décès de la Suisse alémanique. 
annonces sont cependant très rares - trois exemples dans le corpus français, aucun dans le corpus espagnol.

L'exemple suivant illustre en quelque sorte le prototype du faire-part de décès espagnol:

(1) Faire-part de décès espagnol

\section{$+$}

ROGAD A DIOS EN CARIDAD POR EL ALMA SENOR DON NICANO SIMON GARCIA

esposo que fue de la señora

\section{Doña Patroclnio Galindo Martín}

FALLECIO EN SEVILLA EL DIA 27 DE MARZO DE 1994, A LOS OCHENTA Y DOS ANOS DE EDAD, HABIENDO RECIBIDO LOS SANTOS SACRAMENTOS Y LA BENDICION DE SU SANTIDAD R. I. P.

Su esposa; hijos, Narciso, Felicitas, Celia, Pilar y Miguel; hijos politicos, Montserrat Suárez Sánchez, José Calle Madruga, José Palomares Martín y M. Teresa Reviriego Collado; nietos y demás familiares y amigos,

RUEGAN una oración por su alma y asistan a la misa de córpore insepulto que por su eterno descanso tendrá lugar hoy lunes, día 28, a las doce horas, en la parroquia de la Magdalena, y su posterior traslado al cementerio de San Fernando, por cuyos actos de caridad cristiana les quedarán agradecidos.

Vivia: Calle Gravina, 33-2.o A. Sevilla.

Le texte frappe d'abord par sa composition typographique. On y rencontre des emblèmes comme la croix et la bordure noire qui confère à l'annonce une apparence plutôt fermée. Il s'agit d'un texte compact qui éveille l'association à une pierre tombale. La croix tout comme le sigle R.I.P tiré de la liturgie catholique laissent supposer que la dimension religieuse y joue un rôle important.

Le modèle français se distingue clairement du modèle espagnol. Voici l'exemple d'un faire-part de décès français:

(2) Faire-part de décès français

Mme Jacques Jérôme,

son épouse,

Alain Jérôme,

Eric Jérôme et son épouse,

ses enfants,

Béatrice, Vincent, Marie

Jérôme, 


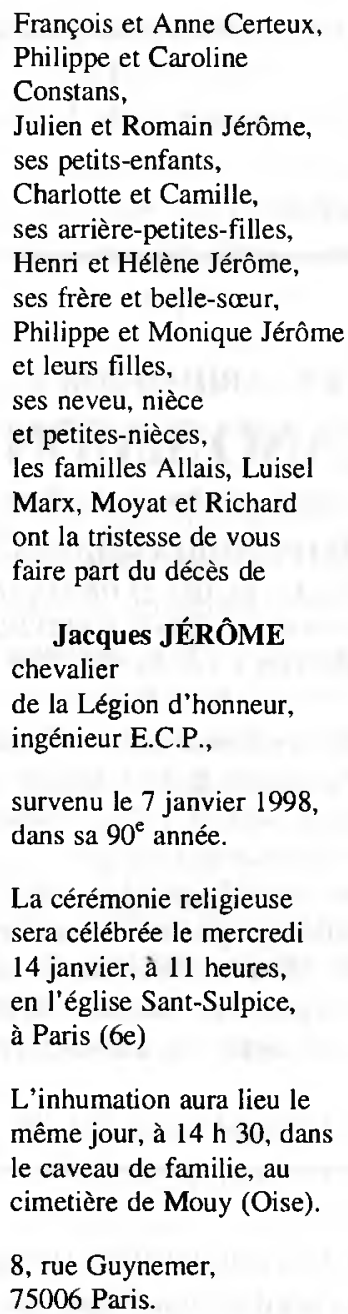

Une comparaison mềme superficielle révèle une mise en page et un agencement du texte tout à fait différents. Le faire-part de décès français se présente comme une petite annonce à l'intérieur d'une colonne. Des symboles comme la croix ou la bordure noire y font défaut. L'usage de caractères gras ou de majuscules est également très restreint. C'est pourquoi, du moins du premier coup d'oeil, le faire-part de décès se distingue à peine d'autres annonces familiales comme l'avis de naissance, etc.

\subsection{MODÈLE DU FAIRE-PART DE DÉCÈS ESPAGNOL}

Pour le faire-part de décès espagnol l'analyse intralinguale donne les composants textuels suivants dont la présentation reflète l'ordre séquentiel typique: 
(3) Composants et ordre séquentiel du faire-part de décès espagnol

[SYMBOLE] - [DICTON, VERSET] - [TITRE D'HONNEUR] - facultatif: [APPEL PRIÈRE 1] - NOM DU DÉFUNT - [ÉTAT CIVIL] - [PROFESSION] - ANNONCE DU DÉCÈS - [LIEU] - DATE - [ÂGE] - [CIRCONSTANCES] - [SACREMENTS] - [BÉNÉDICTION SOUS FORME DE SIGLE] - [FAMILLE, AMIS DANS LE DEUIL] - [APPEL PRIÈRE 2] - [OBSĖQUES] parfois intégré dans [APPEL PRIÈRE 2] - [CONDOLÉANCES] - [DOMICILE DU DÉFUNT]

La liste comporte tous les composants relevés lors de l'analyse du corpus. Trois de ces composants, le nom du défunt, l'annonce du décès et la date, ne manquent dans aucune des annonces examinées. Tous les autres ne se rencontrent que dans une partie des faire-part, ce que j'ai symbolisé ci-dessus par leur mise entre crochets. Pour caractériser l'inventaire des composants textuels il est néanmoins peu convaincant de partir de l'opposition obligatoire vs. facultatif. Il s'agit plutôt de composants plus ou moins fréquents et donc plus ou moins importants aussi. Ici, le concept de prototype semble plus appropriée. Le faire-part de décès prototypique ne contient pas tous les composants énumérés, mais il contient en règle générale plus que les trois composants nucléaires. Un texte qui se limiterait à ces trois composants paraîtrait, au contraire, plutôt déviant par rapport au modèle consacré. Les composants suivants se manifestent dans la plupart des textes examinés et constituent donc en quelque sorte le prototype du faire-part espagnol:

(4) Prototype du faire-part de décès espagnol

SYMBOLE - TITRE D'HONNEUR - facultatif: APPEL PRIÈRE 1 - NOM DU DÉFUNT - ÉTAT CIVIL - ANNONCE DU DÉCĖS - LIEU - DATE - ÂGE SACREMENTS - BÉNÉDICTION SOUS FORME DE SIGLE - FAMILLE, AMIS DANS LE DEUIL - APPEL PRIÈRE 2 - OBSĖQUES

Dans les annonces espagnoles l'ordre séquentiel des composants ne connaît qu'une variation minime. Les différences entre les textes résultent avant tout de la présence vs. absence de certains composants et de leur réalisation verbale. Le tableau (5) ci-dessous présente quelques unes de ces formulations typiques. Je renonce ici à détailler davantage l'aspect formulatif. Dans les paragraphes suivants je me limiterai à commenter les différents composants des faire-part de décès espagnols et à donner - à titre d'exemple - certaines formulations préfabriquées servant à leur réalisation.

(5) Formulations typiques du faire-part de décès espagnol TITRE D’HONNEUR: Ilustrísimo/a Señor/a; parfois abréviation: Ilmo. Señor/llma. Señora

APPEL PRIÈRE 1: $\quad$ Rogad a Dios en Caridad por el alma del señor/de la señora 
ÉTAT CIVIL:

viudo/a de XX; viudo/a que fue de XX; esposo/a que fue de XX

ANNONCE DU DÉCÈS: falleció; descansó en el Señor; fue llamada a la presencia de Dios; ha fallecido

DATE:

ÂGE

SACREMENTS:

el (día) de XX; el pasado XX

a los XX años de edad

habiendo recibido los Santos Sacramentos; habiendo recibido/después de recibir los Santos Sacramentos y la bendición de Su Santidad; confortado/a con los auxilios espirituales

BÉNÉDICTION: $\quad$ D.E.P.; R.I.P.; R.I.P.A.

FAMILLE, AMIS: $\quad$ (selon leur degré de proximité (parentale) au défunt; les noms propres sont facultatifs; s'ils apparaissent, ils viennent toujours après la désignation du lien parental)

APPEL PRIÈRE 2: $\quad$ Ruegan una oración por su alma; Ruegan a sus amistades encomienden su alma a Dios nuestro Señor y ofrezcan una oración por el eterno descanso de su alma, por cuyos actos de caridad cristiana les quedarán agradecidos

OBSÈQUES:

y asistan a la misa que por su eterno descanso se celebrará .../y asistan a la misa de corpore insepulto que tendrá lugar .../y asistan al trasiado de su cadáver que ha de tener lugar .../y asistan a la incineración de su cadáver ...

El funeral por su eterno descanso tendrá lugar .../ El entierro tendrá lugar .../ El entierro partirá .../ La incineración de sus restos mortales tendrá lugar ...

CONDOLÉANCES: el duelo recibe y despide en el citado cementerio/en la citada parroquia

Le composant [TITRE D'HONNEUR], toujours réalisé à l'aide de la formule Ilustrísimo/a señor/a, précède parfois le nom du défunt afin de le valoriser. L' [APPEL à la PRIĖRE 1] se trouve dans la plupart des annonces à la place du titre d'honneur; il est toujours formulé à l'aide d'une structure préfabriquée. Dans cette unité qui invite le lecteur de l'annonce à prier pour le salut de l'âme du défunt, est intégré l'information du décès proprement dite. La formule choisie est toujours Rogad a Dios en caridad por el alma del señor/de la señora. Le composant [ÉTAT CIVIL] se réalise en général à l'aide de l'expression esposo/a que fue de XX ou viudo/a que fue de $X X$, c'est-à-dire qu'il renseigne sur le nom de l'époux ou de l'épouse, même s'il s'agit d'une personne veuve. L'ordre des mots assez inhabituel - contrairement à l'usage le nom est placé avant le pronom que et le verbe - est un indice du caractère figé et stéréotypé de cette tournure. Il s'agit d'un archaîsme maintenu à l'intérieur d'une formule toute faite particulière à ce type de discours spécifique. Le même phénomène est connu en phraséologie où des éléments uniques et formes archaïques sont parfois conservés à l'intérieur d'un phrasème. Ce parallélisme constitue un autre argument 
en faveur du rapprochement proposé entre la phraséologie et la linguistique textuelle, notamment l'analyse des genres discursifs. Il s'avère que tout comme les phrasèmes les genres discursifs plus ou moins figés contribuent à préserver des éléments ou structures archaïques.

Le composant faisant mention des derniers [SACREMENTS] se trouve dans presque toutes les annonces espagnoles. Il souligne, tout comme l'invitation à la prière, la dimension religieuse de ces textes. Pour sa réalisation le locuteur dispose d'un répertoire de formulations apparemment bien restreint comme habiendo recibido los Santos Sacramentos; habiendo recibido/después de recibir los Santos Sacramentos y la bendición de Su Santidad; confortado/a con los auxilios espirituales. La [BÉNÉDICTION] apparat régulierement sous forme de sigle. Fréquentes sont les abréviations D.E.P., R.I.P. et R.I.P.A., toutes originaires de la liturgie catholique. Dans mon corpus il n'y a que deux annonces qui renoncent à l'emploi d'un de ces sigles religieux. L'énumeration des personnes dans le [DEUIL] suit le degré de leur proximité parentale au défunt. Si des noms propres apparaissent, ils précèdent généralement les désignations de rapports familiaux. La liste des personnes dans le deuil forme le sujet d'un énoncé à l'aide duquel le lecteur du faire-part est invité de nouveau à des actes religieux, notamment à des prières. Ce deuxième appel à la [PRIÈRE 2] se fait également à l'aide de formulations très stéréotypées. Les tournures Ruegan una oración por su alma ou Ruegan a sus amistades encomienden su alma a Dios nuesto Señor y orfrezcan una oración por el eterno descanso de su alma ainsi que por cuyos actos de caridad cristiana les quedarán agradecidos servent comme éléments encadrants entre lesquels s'insèrent régulièrement des informations concernant les [OBSÈQUES]. La conjonction que qui introduit la phrase subordonnée, des pronoms et aussi des verbes peuvent être éliminés, ce qui signale de nouveau le caractère préfabriqué de ces structures. Vu que les obsèques ont parfois lieu à plusieurs endroits (église, cimetière), certains faire-part espagnols contiennent également des renseignements précisant où la famille recevra les [CONDOLÉANCES]. La formule consacrée pour la réalisation de ce composant est el duelo recibe y despide en el citado cementeriolen la citada parroquia.

\subsection{MODÈLE DU FAIRE-PART DE DÉCÈS FRANÇAIS}

Le faire-part de décès français contient les composants suivants, dont l'ordre séquentiel semble cependant moins rigide que dans le modèle espagnol:

(6) Composants et ordre séquentiel du faire-part de décès français

[LOCALISATION] - FAMILLE, AMIS DANS LE DEUIL - ANNONCE DU DÉCÈS - NOM DU DÉFUNT - [DÉCORATIONS] - [PROFESSION] - [DATE] - [ÂGE] - [LIEU] - [CIRCONSTANCES] - [OBSÈQUES] - [APPEL AU LECTEUR] - [STATUT COMMUNICATIF DE L'ANNONCE] - [DICTON, VERSET] 


\section{- [COMMÉMORATION DE PROCHES DÉCÉDÉS] - [ADRESSE D'UN/DES MEMBRES DE LA FAMILLE].}

Dans le cas des faire-part de décès français il est plus difficile de déterminer le prototype, car certains de ses composants apparaissent avec une fréquence comparable, mais en des positions séquentielles et des combinaisons différentes. Cela vaut notamment pour les éléments de clôture comme l' [APPEL AU LECTEUR], les informations sur le [STATUT COMMUNICATIF DE L'ANNONCE] ou I'[ADRESSE D'UN/DES MEMBRES DE LA FAMILLE]. En général, les informations concernant les [OBSĖQUES] sont suivies d'au moins un des ces composants encadrants. Il me paraît cependant diffícile d'établir une préférence nette. Le prototype du faire-part de décès français comprend donc les composants suivants:

(7) Prototype du faire-part de décès français

FAMILLE, AMIS DANS LE DEUIL - ANNONCE DU DÉCÈS - NOM DU DÉFUNT - DATE - ÂGE - OBSÈQUES ainsi qu'au moins un composant clôturant.

Les formulations typiques utilisées dans les faire-part français pour la mise en mots des composants les plus fréquents se trouvent rassemblées dans le tableau suivant:

(8) Formulations typiques du faire-part de décès français

LOCALISATION:

FAMILLE, AMIS:

(Renvoi au genre épistolaire)

(selon leur degré de proximité (parentale) au défunt; la désignation du lien parental est facultative; si elle apparaît, elle suit le nom propre. Les femmes mariées n'y figurent souvent que sous le nom de leur mari).

ANNONCE DU DÉCÈS: XX ont la (grande/immense/profonde) douleur/(l'infinie) tristesse/le profond chagrin de (vous) faire part/annoncer le/informer du décès/rappel à Dieu/de la disparition (brutale) de YY; XX (vous) font part du décès de YY.

DATE: survenu le $\mathrm{XX}$

ÂGE:

OBSÈQUES: dans sa $\mathrm{XX}^{\mathrm{e}}$ année/à l'âge de $\mathrm{XX}$ ans

1. Les obsèques ont déjà eu lieu: (Selon sa volonté/son désir) les obsèques (religieuses)/l'inhumation/les funérailles/la cérémonie religieuse/ont eu lieu/a été célébrée (dans la ((plus) stricte) intimité (familiale)), (le XX), (en l'église XX), (à XX).

2. Les obsèques auront lieu: Les obsèques seront célébrées le $\mathrm{XX}$, à $\mathrm{XX}$ heures, en l'église de $\mathrm{XX}$ à $\mathrm{XX}$, suivies de l'inhumation au cimetière de $X X . / L a$ cérémonie religieuse sera célébrée en l'église de XX, le XX, à XX heures. L'inhumation aura lieu le même jour, à XX heures, au cimetière de XX. 
APPEL AU LECTEUR:

Priez pour lui/Des prières et des messes/Ni fleurs ni couronnes/Ni fleurs ni couronnes, des prières (et des messes)

STATUT COMMUNICATIF: Le présent/Cet avis tient lieu de faire-part

Le composant [FAMILLE, AMIS DANS LE DEUIL] se présente également comme une énumération de noms propres agencés selon la proximité du lien parental et/ou amical au défunt. Il constitue le sujet complexe d'un énoncé destiné à informer du décès d'une personne. Les annonces françaises sont donc de véritables avis au public, elles font part au sens propre du mot. Du point de vue linguistique, cela se manifeste par le fait que le composant [ANNONCE DU DÉCÈS] est communiqué à l'aide d'un verbe performatif, lui-même très souvent précédé d'un constituant modalisateur exprimant le deuil ou la douleur. Quant à sa verbalisation on ne relève que peu de variantes: $X X$ ont la (grande/immense/profonde) douleurn'infinie tristesse/le profond chagrin de (vous) faire part/annoncer le/informer du décès/rappel à Dieu/de la disparition (brutale) de $Y Y$, ou simplement: $X X$ (vous) font part du décès de $Y Y$. Quand il s'agit d'un homme, il n'est pas rare que le faire-part de décès français mentionne ses [DÉCORATIONS]. En plus, on rencontre parfois un composant [APPEL AU LECTEUR] l'invitant à des prières ou lui demandant de renoncer à des dons de couronnes et/ou de fleurs. Ces appels sont réalisés à l'aide de formules stéréotypées comme Priez pour lui/Des prières et des messes/Ni fleurs ni couronnes, des prières (et des messes). Une bonne partie des textes comporte un composant métacommunicatif appelé ici [STATUT COMMUNICATIF DE L'ANNONCE]. Il précise que l'annonce constitue le seul moyen de divulgation de l'information et ne connaît que deux formulations très similaires: Le présent/Cet avis tient lieu de faire-part.

Il a déjà été dit que l'ordre séquentiel des composants est moins rigide dans le modèle français que dans le modèle espagnol. Pour les faire-part de décès français on peut distinguer deux modèles séquentiels qui s'opposent surtout dans leur placement du composant [FAMILLE, AMIS DANS LE DEUIL]. Ceci entraîne cependant une composition structurelle différente de toute l'annonce. L'ordre séquentiel reproduit ci-dessus en figure (6) est de loin le plus fréquent. Mais parfois le composant [FAMILLE, AMIS DANS LE DEUIL] suit l' [ANNONCE DU DÉCĖS]. Dans ce cas, on institue d'abord un énonciateur impersonnel qui se charge de la responsabilité communicative de l'acte d'annoncer et qui agit au nom de l'énonciateur réel (on nous prie d'annoncer le rappel à Dieu de ... de la part de). Le pronom impersonnel on renvoie ici au journal dans lequel paraît l'annonce. L'existence de ces variantes montre que la stabilité micro- et macrostructurelle est, même dans le cas d'un type de discours fortement conventionnalisé comme l'est le faire-part de décès, toujours relative. Dans le cas de l'espagnol cela se manifeste seulement dans le choix entre plusieurs formulations préfabriquées pour un même composant pendant que, dans le cas du français, l'ordre séquentiel des composants est également concerné.

Après les deux analyses intralinguales je me propose maintenant de contraster les modèles français et espagnol et de faire ressortir les différences le plus saillantes.

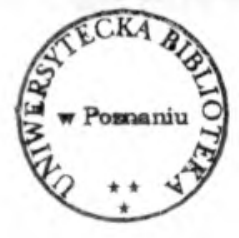




\subsection{ANALYSE CONTRASTIVE DES MODĖLES FRANÇAIS ET ESPAGNOL}

Les différences manifestes dans la mise en page ainsi que dans la composition typographique et emblématique des annonces françaises et espagnoles ont déjà été soulignées dans les paragraphes précédents. L'analyse intralinguale confirme que ces différences dans l'aspect extérieur ne sont point banales, mais renseignent, au contraire, sur des divergences plus fondamentales. Ainsi, au terme de l'analyse contrastive, j'arrive à la conclusion que le faire-part de décès connaît dans chaque communauté linguistique des rapports intertextuels différents, c'est-à-dire qu'il s'est développé à partir de genres communicatifs différents. Dans les annonces françaises étudiées ici manquent et la bénédiction sous forme de sigle et la mention du dernier sacrement. Les deux composants appartiennent cependant au prototype du faire-part de décès espagnol. Dans les textes espagnols l'information du décès est généralement intégrée dans une exhortation à la prière. En plus, les informations concernant les obsèques sont dans la majorité des cas encadrées par des invitations à des actes religieux. La plupart des annonces espagnoles comporte donc à la fois quatre constituants qui accentuent la dimension religieuse. D'un point de vue illocutoire y dominent les actes directifs; grammaticalement cela se manifeste dans la présence d'impératifs (p.ex. Rogad).

Les appels au lecteur, si tant est qu'ils apparaissent dans les annonces françaises, portent sur un autre contenu propositionnel et occupent une autre position séquentielle. Ils sont réalisés de façon très succincte à l'aide d'ellipses qui suppriment justement le verbe à l'impératif. Les différences globales dans la composition du texte trouvent donc leur écho dans la réalisation linguistique de certains de ses composants. Le faire-part de décès français se présente explicitement comme une information et l'affiche généralement par l'utilisation d'un verbe performatif (souvent faire part, parfois aussi annoncer ou informer). Il en résulte des différences concernant la position séquentielle du composant [FAMILLE, AMIS EN DEUIL] qui, dans le modèle français, précède régulièrement l'information du décès proprement dite alors que, dans le modèle espagnol, il est toujours placé après. En plus, dans les textes espagnols, l'acte illocutoire de faire part n'est jamais explicité. Ils affichent plutôt la dimension religieuse en utilisant une multitude de citations chrétiennes et en se donnant dans leur ensemble comme une invitation à la commémoration. Apparemment, cela exclue la présence d'un certain nombre de composants profanes typiques des annonces françaises. Ainsi, le composant métacommunicatif à l'aide duquel on précise le statut communicatif du faire-part n'a pas de contrepartie dans le modèle espagnol. De même manquent les localisations dans l'en-tête de l'annonce que l'on rencontre parfois dans les textes français et qui étayent l'hypothèse selon laquelle ce type de discours est dérivé de formes épistolaires. En plus, la mention des décorations du défunt n'est pas documentée dans le corpus espagnol. Et il est frappant de constater que les manifestations de deuil ou de douleur de la part de la famille du défunt y font complètement défaut. De tels témoignages s'accordent sans doute mal avec l'ancrage évident de la mort dans le système de la foi chrétienne. 
L'analyse contrastive du faire-part de décès montre que, dans les deux communautés linguistico-culturelles opposées ici, ce genre discursif établit des rapports intertextuels assez différents. Dans les annonces françaises, ils sont plutôt profanes. Les annonces espagnoles, par contre, traduisent une forte influence religieuse. Cela est d'autant plus surprenant que les deux pays sont nettement marqués par le catholicisme. Les différences s'expliquent peut-être par le fait que les annonces françaises participent davantage à une tradition laîque républicaine. Vues dans leur ensemble, ces différences me paraissent si importantes qu'elles incitent à réfléchir de nouveau sur l'équivalence communicative des faire-part français et espagnols - équivalence, je le rappelle, postulée comme principe heuristique au début de l'analyse. Surtout lorsqu'on définit les types de discours essentiellement à partir de leur fonction communicative, une telle mise en question me paraît tout à fait pertinente. Les résultats de l'analyse contrastive pourraient alors mener à l'hypothèse que les faire-part de décès français et espagnol ne constituent pas un même genre discursif.

\section{CONCLUSION}

Je coupe court à la comparaison des modèles français et espagnol pour revenir, en guise de conclusion, aux apports théoriques et méthodologiques d'une analyse contrastive de types de discours. À mon avis, ces apports se situent à trois niveaux:

Premièrement, les résultats de l'analyse montrent que l'étude contrastive de genres discursifs soulève des questions méthodologiques qui touchent aux fondements théoriques d'une typologie de textes. Cela vaut notamment pour le choix de critères classificatoires. Il s'est avéré que la composition structuro-formulative spécifique à chaque type de discours y joue un rôle primordial et affecte également l'attribution d'une fonction communicative dominante.

Deuxièmement, les études contrastives font ressortir la spécificité culturelle des genres discursifs. Cet aspect serait à approfondir à l'aide de recherches supplémentaires en linguistique textuelle, mais aussi par des travaux pluridisciplinaires. La linguistique textuelle devrait avant tout s'intéresser aux différentes variantes régionales à l'intérieur d'une communauté linguistique. Les réalisations divergentes dans des exemples mexicains, suisses, belges et franco-canadiens signalent l'importance de cette dimension. En plus, dans le cas particulier du faire-part de décès, son caractère fortement conventionnalisé ne dépend certes pas seulement de la récurrence d'une même tâche communicative, mais aussi de la présence d'un problème affectif. Ici, le recours à des structures figées, voire préfabriquées, permet au locuteur une meilleure gestion de ses émotions. Ces structures facilitent le masquage d'émotions puisque l'individu peut se cacher derrière des rôles sociaux bien définis.

Par ailleurs, ces différences dans la composition des faire-part de décès français et espagnol permettent de conclure d'une manière encore tout à fait provisoire à 
l'existence de cultures du deuil différentes ainsi qu'à la présence, dans chaque société, d'une attitude différente face à un sujet fortement tabouisé comme la mort. Ces différences découlent non seulement des propriétés structuro-linguistiques des annonces, mais aussi du contenu de certains composants, par exemple des informations concernant les obsèques. Ici, un premier point de départ pour des recherches pluridisciplinaires se dégage nettement. Pour l'exemple du faire-part de décès, l'étude d'Ariès (1977) sur L'homme devant la mort, où l'auteur fait ressortir la dimension historique de nos conventions et comportements face à cette question, pourrait ouvrir des horizons nouveaux.

Troisièmement, les analyses contrastives et empiriques de genres discursifs font toujours défaut. Cela vaut notamment pour la linguistique romane où la description de types de discours vient tout juste de s'établir comme champ de recherche. Les études contrastives qui examinent les genres discursifs sous l'angle de leurs spécificités linguistiques et culturelles y sont encore dans leurs débuts. Pourtant, leur nécessité a souvent été signalée par la traductologie, par la didactique des langues et par des travaux en langues de spécialité. Les applications possibles des recherches en 'textologie contrastive' paraissent donc évidentes.

\section{BIBLIOGRAPHIE}

Antos, G. (1982), Grundlagen einer Theorie des Formulierens. Textherstellung in geschriebener und gesprochener Sprache, Tübingen.

A riès, Ph. (1977), L'homme devant la mort, Paris.

Coulmas, F. (1981), Routine im Gespräch: zur pragmatischen Fundierung der Idiomatik, Wiesbaden.

Gülich, E. (1997), Routineformeln und Formulierungsroutinen, dans: Wimmer, Rainer, Franz-Josef Berens (éds), Wortbildung und Phraseologie, Tübingen, 131-175 (communication de 1988).

Lag e-Müll er von der, K. (1995), Text und Tod. Eine handlungstheoretisch orientierte Textsortenbeschreibung am Beispiel der Todesanzeige in der deutschsprachigen Schweiz, Tübingen.

Reiß, K. (1977/1978), Textsortenkonventionen. Vergleichende Untersuchung zur Todesanzeige, Le langage et l'homme 35 et $36,46-54$ et $60-68$.

Spillner, B. (1981), Textsorten im Sprachvergleich. Ansätze zu einer kontrastiven Textologie, dans: Kühlwein, Wolfgang, Gisela Thome, Wolfram Wilss (éds), Kontrastive Linguistik und Übersetzungswissenschaft, München, 239-250.

Stein, S. (1995), Formelhafte Sprache. Untersuchungen zu ihren pragmatischen und kognitiven Funktionen im gegenwärtigen Deutsch, Frankfurt/Main. 